\title{
Aspectos histopatológicos da hiperplasia prostática benigna em cães*
}

\section{Histopathological aspects of canine benign prostatic hyperplasia*}

\author{
Renée Laufer Amorim, ${ }^{* *}$ Enio Pedone Bandarra, ${ }^{* *}$ Veridiana Maria Brianezi Dignani de Moura, ${ }^{* *}$ Giovana Wingeter di Santis ${ }^{* *}$
}

\begin{abstract}
Resumo
O presente estudo consistiu da avaliação histológica de 75 próstatas de cães com idade entre cinco e 15 anos, sendo 41 destas provenientes de material de arquivo e 34 coletadas em necrópsias. Entre as glândulas estudadas, $81,3 \%$ apresentaram hiperplasia, sendo a estromal a de maior freqüência (31,1\%). A associação deste tipo de hiperplasia com atrofia glandular e infiltrado inflamatório se fez presente em cinco animais (26,3\%). A ocorrência concomitante de mais de um tipo de hiperplasia foi observada em $42,6 \%$ dos casos. Animais com hiperplasia epitelial papilífera apresentaram a menor média de idade $(7,1$ anos) entre os tipos histológicos avaliados, enquanto a maior (11,6 anos) foi encontrada em animais com hiperplasia epitelial papilífera e cística concomitante. Os resultados obtidos enfatizam a alta incidência de hiperplasia prostática em cães adultos e idosos, bem como o predomínio do tipo estromal.
\end{abstract}

Palavras-chave: hiperplasia prostática benigna, cães, histopatologia.

\begin{abstract}
In the present study, 75 prostate glands from dogs between five and fifteen years old were studied. 41 were from the Archives of the Pathology Service and 34 collected during necropsy. 81,3\% of the glands had some kind of prostatic hyperplasia. Stromal hyperplasia was the most common (31,1\%). Association between this kind of hyperplasia, glandular atrophy and inflammatory cells were seen in $26,3 \%$ of the animals ( 5 dogs). In $42,6 \%$ of the cases there were more than one kind of prostatic hyperplasia at the same time. Dogs with epithelial prostatic hyperplasia had the lower mean age (7.1 years old) and the highest mean was found in dogs with cystic and epithelial hyperplasia at the same time (11,6 years old). These results showed the high incidence of prostatic hyperplasia in adult and elder dogs, and the prevalence of the stromal component in the hyperplasia.
\end{abstract}

Keywords: benign prostatic hyperplasia, dogs, histopathology.

\section{Introdução}

A hiperplasia prostática benigna (HPB) é um aumento benigno e espontâneo da próstata, observado em cães de meiaidade e idosos (Bamberg-Thalén e Linde-Forsberg, 1992). É a afecção mais comum da próstata canina, uma vez que 100\% dos cães não castrados desenvolvem evidências histológicas de HPB, com o avanço da idade (Barsanti, 1999).

De acordo com Barsanti (1999), a maioria dos animais acometidos não apresenta sinais clínicos, mas em casos sintomáticos, o mais freqüente é o corrimento hemorrágico uretral. A próstata hiperplásica pode variar de pequena e fibromuscular a uma glândula grande com áreas císticas (Weijers et al., 1997). Nos casos mais graves, o aumento prostático pode resultar em estenose uretral, retenção urinária, cistite, glomerulonefrite e hidronefrose (Jubb et al., 1993).

Não é claro se ocorre aumento do número, tamanho das células prostáticas ou ambos nas HPB (Johnston et al., 2000). $\mathrm{Na}$ espécie canina, ao contrário da humana, a hiperplasia é difusa e primária do componente glandular (Poulet, 1985; Barsanti e Finco, 1989); no entanto, há estudos que indicam que hiperplasia ocorre tanto no componente epitelial quanto estromal da glândula de cães idosos (Olson et al., 1987; Laroque et al., 1995).

Observações em microscopia óptica indicaram que a hiperplasia prostática no cão é causada primariamente pela proliferação e hipertrofia das células epiteliais que recobrem os ácinos, bem como proliferação do estroma (Zirkin e Strandberg, 1984). A HPB nos cães pode ser de origem epitelial papilífera ou glandular cística e o componente estromal é observado em quantidades variáveis (Laroque et al., 1995).

Por outro lado, a análise morfométrica do tecido prostático de cães da raça Beagle, feita por Lowseth et al. (1990) indica que, similar às alterações observadas em humanos, a prostatomegalia se deve ao aumento do tecido intersticial e que o componente epitelial não contribui para o aumento observado, enfatizando o uso do cão como modelo desta doença no homem.

\footnotetext{
1 Projeto financiado pela FAPESP

** Faculdade de Medicina Veterinária e Zootecnia, UNESP, Botucatu.

Serviço de Patologia Veterinária - Departamento de Clínica Veterinária - FMVZ, Unesp, Botucatu, SP - CEP 18618-000 - e-mail: renee@fmvz.unesp.br
} 
Diante da alta freqüência desta afecção em cães e das dúvidas que persistem sobre o aspecto histopatológico predominante das HPB, o presente trabalho teve como objetivo classificar e verificar a freqüência dos diferentes tipos de HPB, informações importantes na comparação de estudos entre a espécie canina e humana.

\section{Material e métodos}

Foram analisadas 75 próstatas caninas, sendo 41 provenientes do material de arquivo do Serviço de Patologia Veterinária da FMVZ - UNESP - Botucatu, SP e 34 novas amostras coletadas nas necrópsias da rotina do mesmo Serviço.

Para o estudo retrospectivo foram selecionados os casos dos últimos dez anos em que havia relato de alteração prostática (hipertrofia, hiperplasia, prostatite, abscessos, neoplasias). No estudo prospectivo foram coletadas em solução de formol tamponado a 10\%, de forma aleatória, próstata de 34 caninos encaminhados ao Serviço de Patologia Veterinária para exame necroscópico. A idade dos animais variou entre cinco e 15 anos.

O material foi incluído em parafina e cortes de $4 \mu \mathrm{m}$ foram obtidos em micrótomo automático, distendidos em lâminas histológicas e corados pela coloração de H\&E. As lâminas foram analisadas em microscópio óptico e instituído um diagnóstico final, de acordo com os achados histopatológicos.

\section{Resultados}

Das próstatas estudadas, 61 (81,3\%) apresentaram algum tipo de hiperplasia, sendo a estromal a de maior freqüência, observada em 19 animais (31,1\%). A ocorrência das demais afecções está ilustrada na Figura 1.

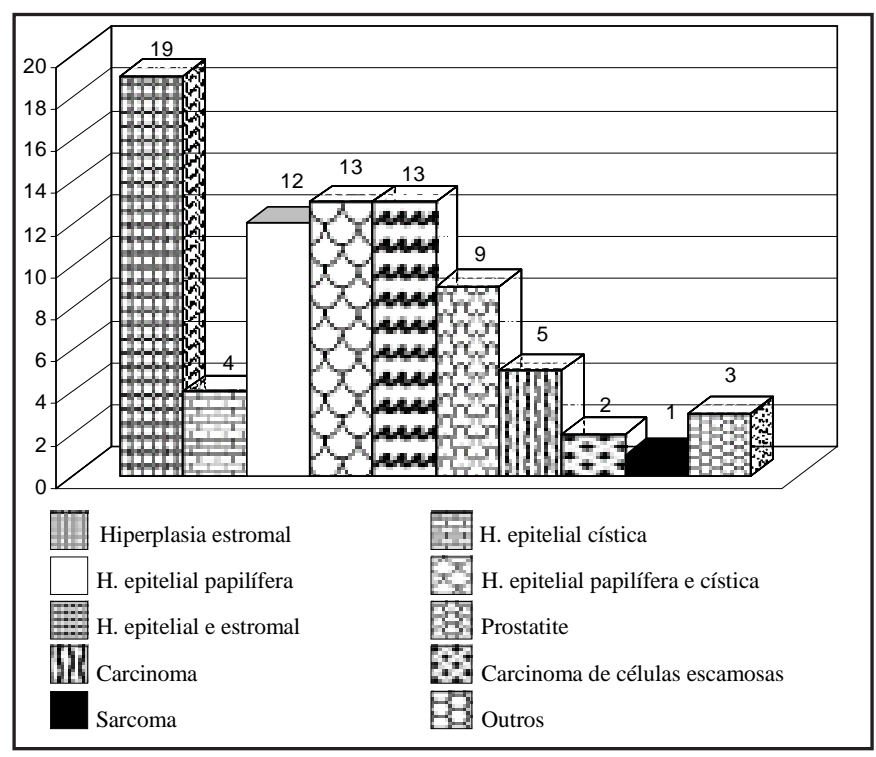

Figura 1 - Diagnósticos histopatológicos instituídos nas glândulas avaliadas $(n=75)$. Notar que o número total de diagnósticos ultrapassa o número total de animais devido à ocorrência de lesões concomitantes.

O diagnóstico de hiperplasia estromal foi instituído com base na proliferação do estroma fibroso ou muscular, associado ou não à atrofia glandular e presença de infiltrado inflamató- rio intersticial mononuclear (Figura 2). Tal concomitância se fez presente em cinco $(26,3 \%)$ casos de hiperplasia estromal.

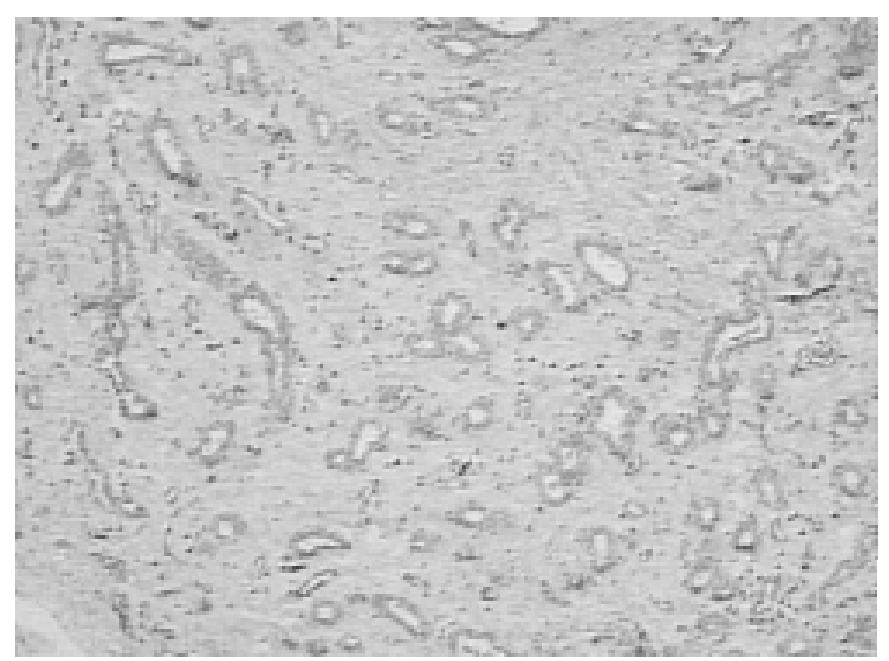

Figura 2 - Fotomicrografia de próstata canina - hiperplasia estromal. Observam-se proliferação estromal e atrofia glandular. HE/200x

A forma epitelial papilífera se caracterizou pela hiperplasia digitiforme, para o interior da luz glandular, das células epiteliais, mantendo a membrana basal intacta (Figura 3).

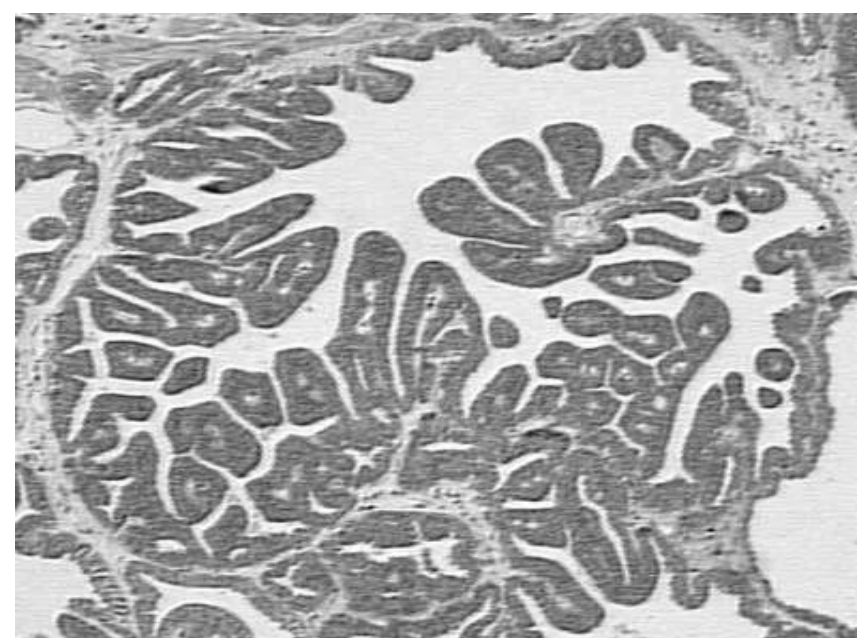

Figura 3 - Fotomicrografia de próstata canina - hiperplasia epitelial papilífera. Ácino hipercelular com projeções papilíferas intraluminais recobertas por várias camadas de epitélio cilíndrico de aspecto benigno. $\mathrm{HE} / 100 \mathrm{x}$.

A presença de ácinos dilatados, de tamanhos irregulares, arredondados e recobertos por epitélio achatado, com ou sem secreção luminal, determinou o diagnóstico de hiperplasia epitelial cística (Figura 4). Em alguns casos a glândula cística mostrava projeções papilíferas para a luz do ácino.

Em 26 (42,6\%) animais foi diagnosticada a ocorrência concomitante de mais de um tipo de hiperplasia, havendo associações de hiperplasia epitelial papilífera e cística e de hiperplasia estromal com algum dos tipos de hiperplasia epitelial (papilífera ou cística) (Figura 5). 
A média de idade dos animais com hiperplasia estromal foi de 8,1 anos. Aqueles que apresentavam hiperplasia epitelial papilífera e cística, apresentaram média de 7,1 e 10,7 anos, respectivamente. A associação destes dois tipos de hiperplasia ocorreu em cães com idade média de 11,6 anos. A idade média de ocorrência concomitante de hiperplasia estromal e papilífera foi de 7,6 anos (Figura 6).

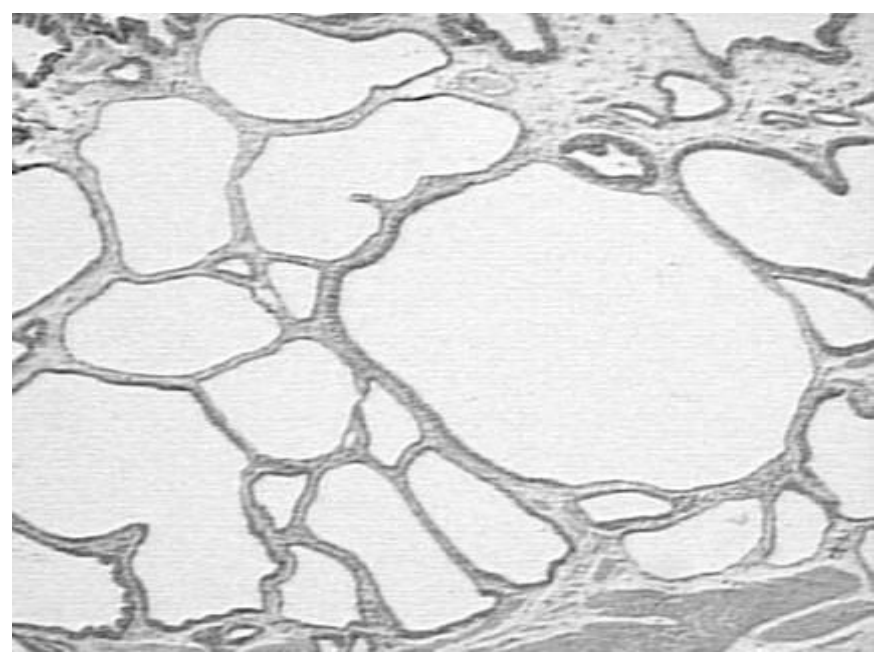

Figura 4 - Fotomicrografia de próstata canina - hiperplasia cística glandular. Ácinos dilatados recobertos por epitélio cúbico simples sem evidências de atividade secretora. HE 100x.

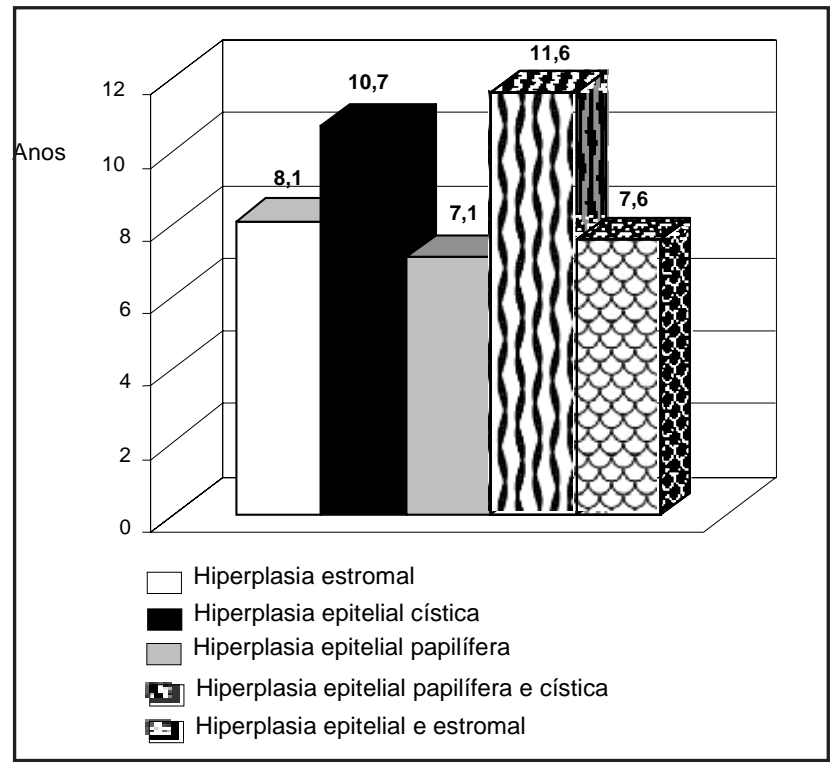

Figura 6 - Média de idade dos animais acometidos pelos diferentes tipos de hiperplasia, de forma simples ou associada.

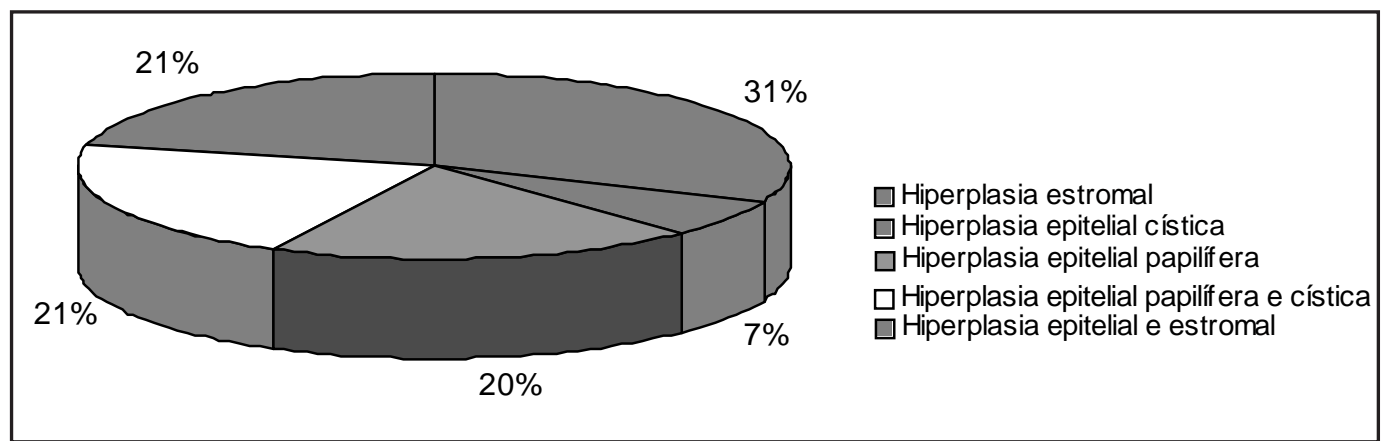

Figura 5 - Ocorrência dos diferentes tipos histológicos de hiperplasia e suas associações nas próstatas caninas avaliadas $(\mathrm{n}=61)$.

\section{Discussão e conclusões}

A freqüência de HPB $(81,3 \%)$ encontrada neste trabalho está de acordo com Chew (1997), que descreve a hiperplasia como a desordem prostática canina mais comum, seguida por prostatite e neoplasia. Cartee e Rowles (1983) também relatam a maior ocorrência de HPB e, em seguida, os abscessos.

A maior freqüência de hiperplasia estromal observada neste estudo $(31,1 \%)$ está de acordo com a literatura humana, a qual afirma que esta afecção tem origem no estroma da glândula prostática (Shapiro et al., 1992ab; Ishigooka et al., 1996; Kurimoto et al., 1998). O diagnóstico de hiperplasia estromal foi caracterizado pela proliferação do estroma fibroso ou muscular, associado ou não a atrofia glandular e presença de infiltrado inflamatório intersticial mononuclear. Segundo Lee et al. (1997), células inflamatórias como macrófagos e linfócitos secretam interleucinas capazes de estimular a síntese de colágeno e, dessa forma, auxiliam na proliferação do componente estromal. Assim, a hiperplasia estromal pode ser secundária à presença dessas células inflamatórias, e a atrofia glandular seria conseqüência da proliferação do estroma.

Em cães que não apresentam afecções prostáticas, há predomínio do componente estromal antes da maturidade sexual e, após este período, o epitélio glandular prevalece (Zirkin e Strandberg, 1984; Barsanti e Finco, 1989). Com base neste dado, a observação da maior quantidade de estroma na próstata de cães acima de cinco anos (que já atingiram a maturidade sexual), permitiu o diagnóstico de hiperplasia estromal, e não de um processo fisiológico, que ocorre com o avançar da idade. 
No presente estudo, parte dos casos de HPB epitelial apresentou uma concomitância entre as formas papilífera e cística. A ocorrência desta associação está de acordo com Laroque et al. (1995) que citam que a HPB nos cães pode ser epitelial papilífera ou cística, bem como haver uma interação entre as formas.

Como foi demonstrado, além da presença concomitante de hiperplasia epitelial papilífera e cística, também foi observada a ocorrência de hiperplasia estromal e algum dos tipos de hiperplasia epitelial. No entanto, estudos sobre hiperplasia em cães apresentam dados controversos, havendo autores que relatam a gênese da HPB na proliferação de células do componente epitelial (Poulet, 1985; Barsanti e Finco, 1989), alguns que descrevem a hiperplasia estromal como responsável pelo processo (Lowseth et al., 1990; Klausner et al., 1994), e outros afirmam que esta afecção é de origem epitelial e estromal (Zirkin e Strandberg, 1984; Laroque et al., 1995).

No trabalho de Klausner et al. (1994), animais acima de cinco anos de idade apresentaram hiperplasia estromal com atrofia acinar e infiltrado inflamatório mononuclear. Este achado concorda com o dados obtidos no presente estudo, onde $26,3 \%$ dos animais apresentavam atrofia glandular e infiltrado inflamatório mononuclear associados à hiperplasia estromal.

Em relação à idade dos animais com HPB, Barsanti (1999) descreve que $100 \%$ dos cães não castrados desenvolvem evidências histológicas de HPB com o avanço da idade. Zirkin e Strandberg (1984) relatam que a média de idade entre os cães com HPB é de 6,4 anos, embora a maioria dos autores concorde que algum grau de hiperplasia é evidente em animais acima de quatro anos e a incidência aumenta com o avançar da idade, sendo que cerca de $80 \%$ dos cães adultos e idosos possuem próstatas aumentadas (Barsanti e Finco, 1989; Jubb et al., 1993; Johnston et al., 2000). Todos os cães com HPB deste estudo eram adultos ou idosos.

\section{Referências}

AQUILINA, J.W.;MCKINNEY, L.A.;PACELLI, A.; RICHMAN, L.K.;WATERS, D.J.; THOMPSON, I.; BURGHARDT JR., W.F.; BOSTWICK, D.G. High grade prostatic intraepithelial neoplasia in military working dogs with and without prostate cancer. The Prostate. v. 36, n. 3, p. 189-193, 1998.

BAMBERG-THALÉN, B.; LINDE-FORSBERG, C. The effects of medroxyprogesterone acetate and ethinylestradiol on hemogram, prostate, testes and semen quality in normal dogs. Journal of the American Veterinary Medical Association, v. 39, n. 4, p. 264-270, 1992.

BARSANTI, J.A. Prostatic hyperplasia: medical therapy. Proceedings of the American Clinical Veterinary Internal Medicine, Chicago, v. 17, p. 536-538, 1999.

BARSANTI, J.A.; FINCO, D.R. Canine prostatic diseases. In: ETTINGER S.J. Textbook of veterinary internal medicine. 3. ed. Philadelphia: Saunders, 1989. v. 2, p. 1859-1880.

CARTEE, R.E., ROWLES, T. Transabidominal sonographic evaluation of the canine prostate. Veterinary Radiology, v. 24, n. 4, p. 156-164, 1983.

CHEW, D.J. An overview of prostatic disease. Compendium on Continuing Education for the Practicing Veterinarian. v. 19, n. 3, p. 80-85, 1997.

COTRAN, R.S.; KUMAR, V.; ROBBINS, S.L. The male genital system. In: . Pathological basis of disease. 6. ed. Philadelphia: Saunders, 1999. p. $1025-1033$.
De acordo com Lee et al. (1997), com o avançar da idade, há um aumento do infiltrado inflamatório mononuclear prostático sem a presença de infecção, porém, contribuindo ativamente para o desenvolvimento da HPB devido ao estímulo que essas células inflamatórias exercem na proliferação de colágeno.

Lesões pré-neoplásicas ou precursoras de neoplasias, como as displasias, também chamadas de neoplasia prostática intra-epitelial (PIN), foram observadas com alta freqüência associadas aos adenocarcinomas prostáticos em caninos e humanos (Aquilina et al., 1998; Cotran et al., 1999). Nesta pesquisa foram observadas células displásicas associadas à hiperplasia epitelial papilífera e em glândulas localizadas na região de infiltrado inflamatório do estroma fibromuscular. Os focos de displasia epitelial presentes nas áreas de infiltrado inflamatório podem ser a origem dos processos neoplásicos da próstata, já que são considerados por alguns pesquisadores como PIN (Waters e Brostwick, 1997ab).

Na espécie humana, as hiperplasias não são consideradas lesões precursoras do adenocarcinoma prostático, pois se estabeleceu que a HPB surge da zona transicional da próstata e o câncer da região periférica (Cotran et al., 1999). Nos cães, se as hiperplasias fossem lesões pré-neoplásicas, provavelmente a incidência dos tumores prostáticos seria maior, já que 80 a $100 \%$ dos caninos acima de cinco anos apresentam HPB (Jubb et al., 1993; Barsanti, 1999), o que é demonstrado também neste trabalho, onde se observam alta incidência de HPB e baixa de carcinomas prostáticos (6,7\%).

Diante do exposto, conclui-se que a hiperplasia prostática benigna é a alteração prostática mais freqüente entre os cães adultos e idosos, sendo a HPB estromal a forma de maior ocorrência dentre os diferentes tipos descritos na literatura e observados neste estudo.

ISHIGOOKA, M.; HAYAMI, S.; SUZUKI, Y.; HASHIMOTO, T.; SASAGAWA, I.; NAKADA, T. Age-related changes of histological composition in established benign prostatic hyperplasia. European Urology, v. 29, n. 1, p. 85-89, 1996.

JOHNSTON, S.D.; KAMOLPATANA, K.; ROOT-KUSTRITZ, M.V.; JOHNSTON, G.R. Prostatic disorders in the dog. Animal Reproduction Science, v. 60, n. 61, p. 405-415, 2000.

JUBB, K.V.F.; KENNEDY, P.C.; PALMER, M. Male reproductive system. In:__. Pathology of domestic animals, 4. ed. San Diego:Academic Press, 1993. v. 3, p. 471-529.

KLAUSNER, J.S.; BELL, F.W.; HAYDEN, D.W.; JOHNSTON, S.D.; LUND, E.M. Recent developments in the diagnosis and treatment of BPH and prostatic carcinoma. Proceedings of American College Veterinary Internal Medicine. p. 547-548, 1994.

KURIMOTO, S.; MORIYAMA, N.; HAMADA, K.; KAWABE, K. Evaluation of histological structure and its effect on the distribution of alpha-1 adrenoreceptors in human benign prostatic hyperplasia. British Journal of Urology, v. 81, n. 3, p. 388-393, 1998.

LAROQUE, P.A.; PRAHALADA, S.; MOLON-NOBLOT, S.; COHEN, S.M.; SOPER, K.; DUPRAT, P.; PETER, C.P.; VANZWIETEN, M.J. Quantitative evaluation of glandular and stromal compartments in hyperplastic dog prostates: effect of 5-alpha reductase inhibitors. The Prostate, v. 27 , n. 3, p. 121-128, 1995.

LEE, C.; KOZLOWSKI, J.M.; GRAYHACK, J.T. Intrinsic and extrinsic factors controlling benign prostatic growth. The Prostate, v. 31, n. 2, p. 131-138, 1997. 
LOWSETH, L.A.; GERLACH, R.F.; GILLETT, N.A.; MUGGENBURG, B.A. Age-related in the prostate and testes of the beagle dog. Veterinary Pathology, v. 27, n. 5, p. 347-353, 1990.

OLSON, P.N.; WRIGLEY, R.H.; THRALL, M.A.; HUSTED, P.W. Disorders of the canine prostate gland: pathogenesis, diagnosis and medical therapy. Compendium on Continuing Education for the Practicing Veterinarian, v. 9, n. 6, p. 613-624, 1987.

POULET, F. Evolution de la prostate canine en fonction de l'âge. Annales de Medecine Veterinaire, v. 129, n. 8, p. 567-584, 1985.

SHAPIRO, E.; HARTANTO, V.; LEPOR, H. Quantifying the smooth muscle content of the prostate using double-immunoenzymatic staining and color assisted image analyses. The Journal of Urology, v. 147, n. 4, p.1167-1170, 1992a.

SHAPIRO, E.; BECICH, M.J.; HARTANTO, V.; LEPOR, $\mathrm{H}$. The relative proportin of stromal and epithelial hyperplasia is related to the development of symptomatic benign prostate hyperplasia. The Journal of Urology. v. 147, n. 5, p. 1293-1297, 1992b.

WATERS, D.J.;BOSTWICK, D.G. The canine prostate is a spontaneous model of intraepithelial neoplasia and prostate cancer progression. Anticancer Research, v. 17, n. 3A, p. 1467-1470, 1997a.

WATERS, D.J., BOSTWICK, D.G. Prostatic intraepithelial neoplasia occurs spontaneously in the canine prostate. The Journal of Urology, v. 157, n. 2, p. 713-716, 1997b.

WEIJERS, R.E.;ZAMBON, J.V.; KESSELS, A.G.H.;BRÜINE, A.P. On the prediction of the histological composition of benign prostatic hyperplasia based on clinical and MRI parameters. The Prostate, v. 32, n. 3, p. 179187, 1997.

ZIRKIN, B.R., STRANDBERG, J.D. Quantitative changes in the morphology of the aging canine prostate. The Anatomical Record, v. 208, n. 2, p. 207-214, 1984. 